\title{
Intraoperative Feedback and Quality Control in Orbital Reconstruction
}

\section{The Past, the Present, and the Future}

\author{
Ruud Schreurs, MSc ${ }^{a, b, *}$, Frank Wilde, MD, DDS ${ }^{c, d}$, Alexander Schramm, MD, DDS ${ }^{c, d}$, \\ Nils-Claudius Gellrich, MD, DDS ${ }^{e}$
}

\begin{abstract}
KEYWORDS
- Computer-assisted surgery • Orbit • Orbital reconstruction • Surgical navigation • Intraoperative imaging • Patient-specific implants • Intraoperative feedback • Quality control

KEY POINTS

- The development and implementation of medical technology in orbital reconstructive surgery is a logical consequence of the philosophy to optimize the treatment procedure before, during, and after surgery in terms of maximum control for the surgeon and the aim for optimal outcome for the individual patient.

- During the surgical procedure, the optimal virtual surgical planning must be transferred to the surgical site as accurately as possible; for this purpose, a variety of different methods and devices may be used and even combined, depending on the complexity of the task.

- The adage that "the best way to predict the future is to invent it" (Alan Kay) has proved true for orbital reconstruction in the past and will be the key to future innovations.
\end{abstract}

\section{Introduction: the rationale of technology in orbital surgery}

Over the last 20 years, rapid changes in digital medical technology have occurred if it comes to "making the invisible visible." Especially in craniomaxillofacial surgery, complex reconstructive procedures in primary or secondary cases have always been a challenge in the past and will remain as such in the future. Patients have a different view on their pathology than the surgeon has: the patient has an outside-oriented perspective, which relates to outer appearance and appropriate function. The surgeon has to take the outer tissue envelope into consideration to focus on the inside-oriented perspective, which is the craniofacial skeleton, and the associated soft tissues to analyze the amount of individual

\footnotetext{
a Department of Oral and Maxillofacial Surgery, Amsterdam UMC Location AMC and Academic Center for Dentistry Amsterdam (ACTA), Meibergdreef 9, 1105 AZ Amsterdam, the Netherlands

${ }^{b}$ Department of Oral and Maxillofacial Surgery, Radboud University Medical Centre Nijmegen, Geert Grooteplein Zuid 10, Nijmegen 6525 $\mathrm{GA}$, the Netherlands

c Department of Oral, Maxillofacial and Plastic Surgery, German Armed Forces Hospital of Ulm, Oberer Eselsberg 40, Ulm 89081, Germany

d Department of Oral and Maxillofacial Surgery, University Hospital, Ulm University, Albert-Einstein-Allee 23, Ulm 89081, Germany

e Department of Oral and Maxillofacial Surgery, Hannover Medical School, Carl-Neuberg-Str. 1, Hannover 30625, Germany

* Corresponding author.

E-mail address: r.schreurs@amsterdamumc.nl
}

deformity, to modify these, and to finally achieve an appropriate esthetic result for that patient combined with adequate function.

Detailed knowledge of the anatomic structures is fundamental for orbital surgery: inadequate reconstructions potentially lead to unsatisfactory results. ${ }^{1}$ For this reason, clinical assessment, combined with imaging techniques used for diagnosis and treatment planning, must meet the highest standards. It is only then possible to answer the most important question in orbital fracture management: do we have to explore an orbital defect? This ongoing debate is further discussed in Leander Dubois and colleagues' article,"Ongoing Debate in Clinical Decision Making in Orbital Fractures: Indications, Timing, and Biomaterials," in this issue. If surgery is indicated, it is the task of the surgeon to minimize the risk for the patient and achieve the adequate result, which brings the following questions: do we have a plan for our intended result? Is this plan achievable? Is the plan adequate? Is the plan available during surgery? Can feedback on the plan be obtained intraoperatively? Did we achieve the intended result?

These questions led to the introduction and implementation of computer-assisted surgery. From this perspective, all phases of treatment (before, during, and after the surgical procedure) should be optimized for the individual patient. Medical technology turned out to be the cornerstone to take this idea to reality: with technological developments, the limitations in orbital reconstruction could be overcome and the predictability of the results improved. This article covers the intraoperative phase of computer-assisted surgery; with the "why" provided, focus is on the past developments that led to 

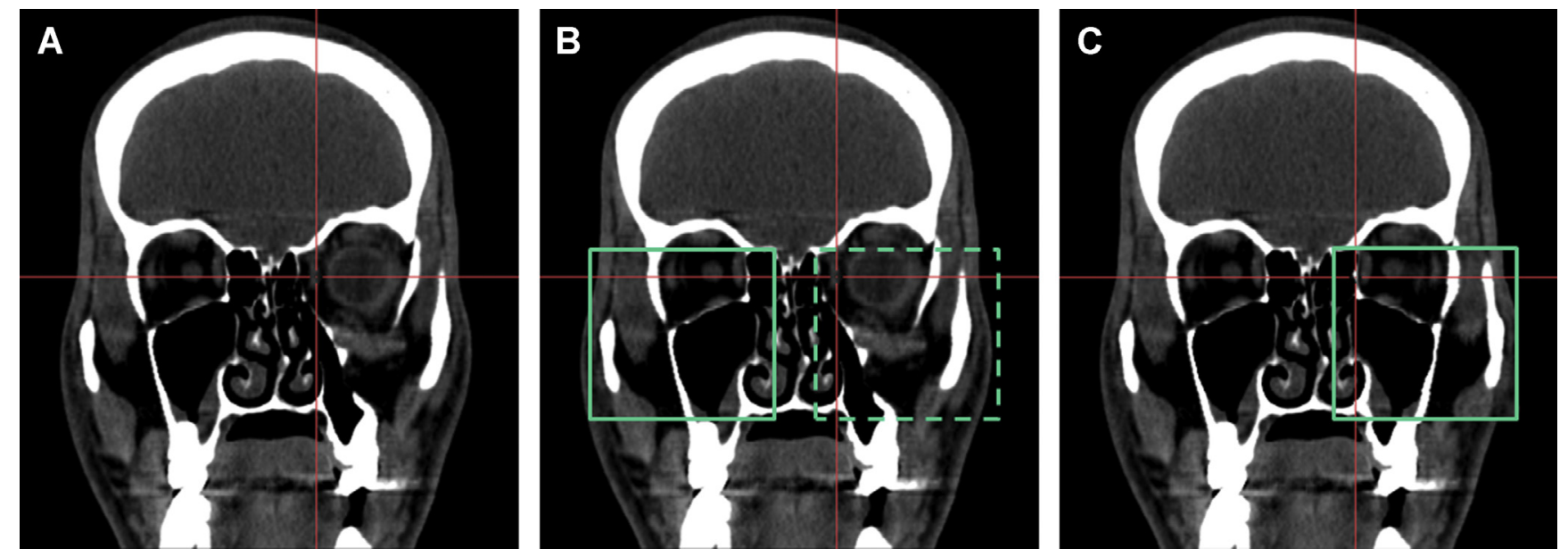

Fig. 1 Visualization of an early virtual surgical planning for an orbitozygomatic complex fracture. A volume of interest is indicated on the unaffected side $(A)$ and mirrored to the affected side $(B)$, effectively generating a secondary DICOM dataset with the desired reconstruction $(C)$. Only the coronal view is shown here for simplification, but all multiplanar directions are available to the surgeon. (Courtesy of Ruud Schreurs, MSc, Frank Wilde, MD, DDS, Alexander Schramm, MD, DDS, and Nils-Claudius Gellrich, MD, DDS.)

intraoperative control, the current state of the art, and the possible future of feedback and quality control in orbital surgery: the how, the now, and the wow.

\section{How: the history of intraoperative feedback}

Historically, several 2-dimensional (2D) radiographs had been used to approach orbital deformity correction. Today none of these have any impact anymore, and-by the way-there never could be a plan nor a control to what was loaded in and on the orbit. Surgery of the orbit based on 2D radiographs must have been a walk in the mist and darkness. The headway to 3D imaging, in case of orbital reconstruction helical and conebeam computed tomography (CBCT) (only in selected cases combined with $M R I)$, meets the requirement to treat a $3 D$ defect with the aid of a 3D data set. However, in the eighties of the last decade these data were only used to look at, which meant that trying to understand the individual patient's deformity depended on the views selected by the radiologist. From the nineties onwards, computer technology allowed the surgeon to interactively use the volume data sets on independent analyzing platforms. Clinicians became able to modify the individual volume data set to the intended result.

Mirroring the unaffected side onto the deformed side was the first attempt to virtually correct the bony framework and create a digital blueprint for the reconstruction in unilateral craniofacial defects (Fig. 1). This technique was developed and taken into routine by the Freiburg group of Rainer Schmelzeisen, Alexander Schramm, and Nils-Claudius Gellrich, in cooperation with the former Leibinger Company, trying to make the STN Zeiss/Leibinger navigation system applicable for craniomaxillofacial surgery in those days. ${ }^{2}$ All planning had to be performed on the navigation machine itself; it was only in the early 2000s that medical industry was able to serve the demands of the surgeon, and virtual planning became feasible with software on stand-alone machines, independent of the navigation system. It was the cooperation with Brainlab that
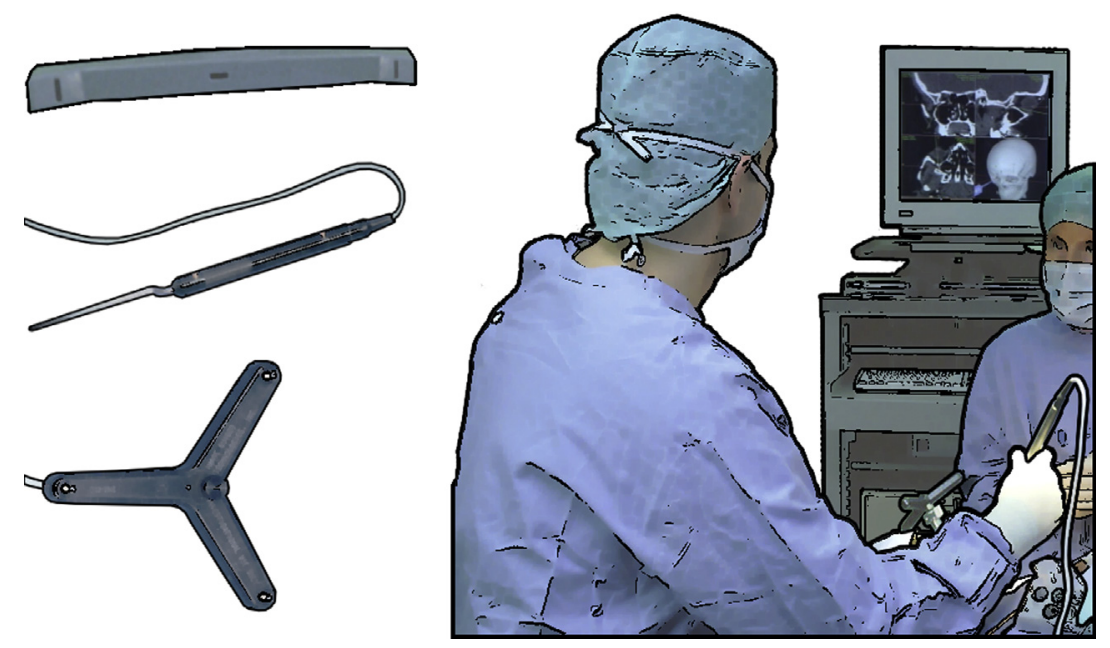

Fig. 2 Instruments of an early navigation system (left) and the use of the navigation system (right). This system was an optical tracking system with active light-emitting diodes (LEDs) on the instrument and reference array, whose signal was captured by the camera in the top left. Currently, passive and wireless optical systems (see Fig. 8) are most frequently used. (Courtesy of Ruud Schreurs, MSc, Frank Wilde, MD, DDS, Alexander Schramm, MD, DDS, and Nils-Claudius Gellrich, MD, DDS.) 

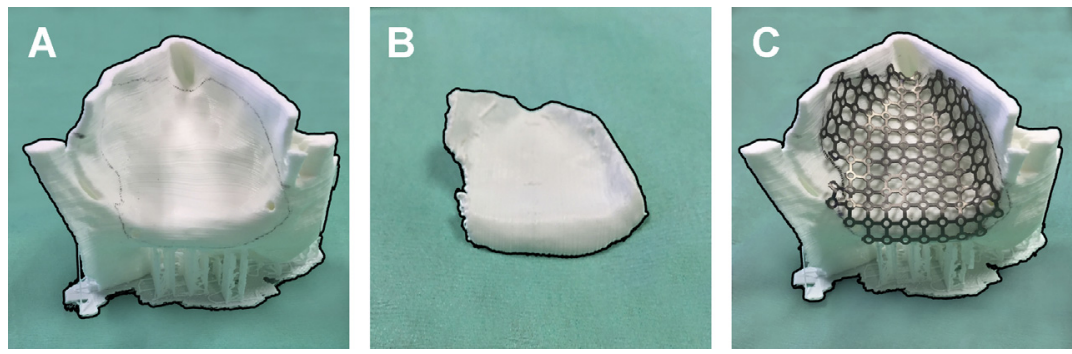

Fig. 3 Prebent implant on an individualized biomodel. In $(A)$, the individualized biomodel is shown, whereas $(B)$ shows the desired shape of the implant, as constructed in the virtual planning software. The result of the prebent implant is shown in (C). (Courtesy of Ruud Schreurs, MSc, Frank Wilde, MD, DDS, Alexander Schramm, MD, DDS, and Nils-Claudius Gellrich, MD, DDS.)

resulted in this advancement in planning logistics and the availability of advanced software tools for virtual planning. ${ }^{3}$

The navigation technology may be viewed as the offspring of frame-based stereotaxy, introduced by Horsley and Clarke in 1908. ${ }^{4-7}$ Their Horsley-Clarke apparatus, of which the first was produced at a cost of $£ 300$, included the use of geometric measurements in a Cartesian coordinate frame. ${ }^{5,6}$ An atlas of the position of brain structures within the coordinate frame was generated for cats and monkeys, and the frame and atlas could even be compensated for different head sizes. ${ }^{5}$ It was not until 1947 that a modified version of the apparatus, proposed by Spiegel and Wycis, was used in human brain surgery. ${ }^{8}$ The link with the preoperative imaging was made with the introduction of frameless stereotaxy in 1987: the position of the pointer tip of an articulating mechanical arm was related to the image data acquired before the operation. ${ }^{7,9}$ Optical tracking of the instrument and patient meant increased freedom of movement and easier use of the navigation system. ${ }^{7}$ The use of navigation in orbital surgery, almost a decade after Horsley and Clarke laid the groundwork, was kick-started with an article describing the use in a group of 18 patients by the Freiburg group. ${ }^{2}$ An image of one of the navigation systems used in these days is provided in Fig. 2.

Developments in $3 \mathrm{D}$ imaging have had another effect next to the development of surgical planning and the utilization during surgical navigation: 3D imaging technology became available in the OR itself. Already in the early 1980s applications of imaging during neurosurgery were described, but the fixed position of the scanner and limited image quality hampered it widespread use. ${ }^{10-13}$ Advances in image quality, image equipment, and mobility of intraoperative CT or CBCT scanners over the years paved the way for utilization within oral and maxillofacial surgery: it is not surprising that reconstruction of orbital defects was among the first applications. ${ }^{14}$

In the meantime, the gap had to be closed between virtual planning, navigation, and intraoperative imaging on one side and the implant to achieve the desired result on the other. Individualization of orbital implants was historically achieved by preshaping whatever biomaterial during surgery and was thereby depending on the experience and skills of the individual surgeon. Deeply respected colleagues such as Beat Hammer, Joe Gruss, Paul Manson, and others had their own way to prebend, or mold on a nonindividual reference model, with outstanding results. But even at that time they already included some kind of reference, whether it was schematic drawings only or the aforementioned reference skull draped with a sterile foil to serve as an aid for molding the reconstruction material. With further development of $3 \mathrm{D}$ printing technologies more and more individualized biomodels of the given (deformed) patient's anatomy or the virtually corrected anatomy became available to shape a nonpreformed biomaterial toward an individual implant, either preoperatively or intraoperatively (Fig. 3). ${ }^{15,16}$ This technique still is a valid and an adequate method, which helps to master nearly all kind of craniofacial deformity correction. Latest technology allows to even provide 3D-printed patient-specific biomodels, which are autoclavable.

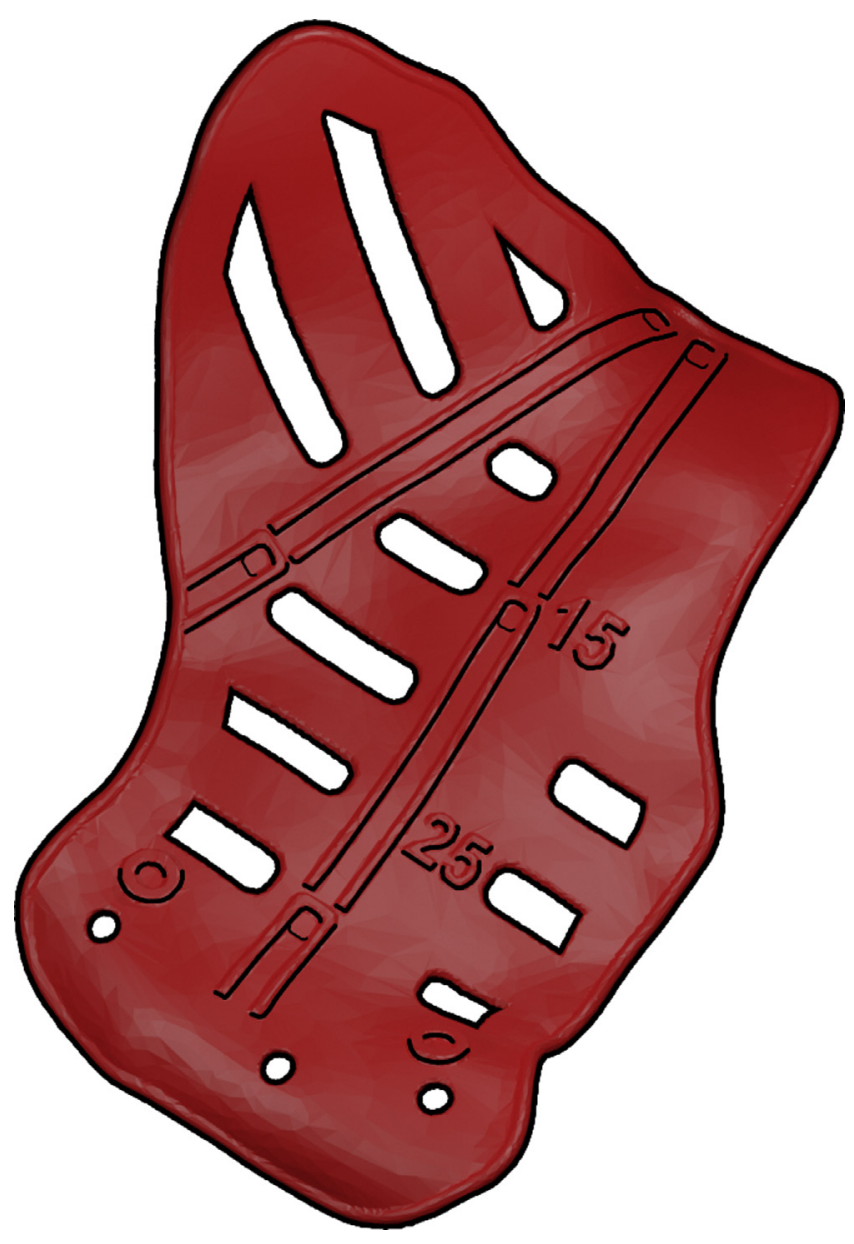

Fig. 4 Patient-specific implant with functionalization and preventive design parameters. The different thickness, cord-style outer boundary, and navigation markers and rulers can be distinguished. (Courtesy of Ruud Schreurs, MSc, Frank Wilde, MD, DDS, Alexander Schramm, MD, DDS, and Nils-Claudius Gellrich, MD, DDS.) 
A different approach to shaping an implant was developed in parallel. Research on the average shape of the orbit performed by Marc Metzger's group (Freiburg) was a preface for the development of a preformed orbital implant based on the average shape of the orbit and possible defects encountered. ${ }^{17-19}$ In theory, the use of such an implant prevents the necessity of preoperative or intraoperative bending altogether, thus saving time and being less dependent on the molding skills of the surgeon. ${ }^{19,20}$ Another advantage lies in the possibility to import a virtual model of the preformed implant in the virtual surgical planning. The optimal position of the implant can be determined based on characteristics of patient and defect; it is even possible to evaluate preformed implants of several sizes or different manufacturers (see also article, "Advanced Diagnostics and Three-dimensional Virtual Surgical Planning in Orbital Reconstruction", in this issue). ${ }^{20-22}$

With emerging skills of analyzing patient's volume data sets, the view of taking an individual virtual plan toward an adequate individual anatomic shaped implant changed the requirements of what an implant should be able to provide and represent: above the effect of an anatomic replacement the terms "functionalization" and "preventive design" of an implant came up. Functionalization implies that functions were added to the implant, for example, position control information was added for navigation purposes. Furthermore, anatomic extensions or flings can be added to an implant, so that the implant properly leavers on reliable structures in the defect site. Preventive design implies that typical mistakes that might occur with specific procedures can be diminished: in reconstructive orbital surgery this refers to change of implant shape, sharp edges, and possible interference with the optic nerve or eye muscles especially in the orbital cone area. This is why the Hannover Group with Majeed Rana and Nils-Claudius Gellrich developed a patient-specific orbital implant with a distinct topography of different thickness, a slightly thickened circular outer rounded cord-style implant border, an inverted snow-shovel design in the area of the posterior ledge, and multiple linear openings perpendicular to the transition zone for the medial orbital wall and perpendicular to the parasagittal oblique vector for the orbital floor. ${ }^{23}$ These implementations can be seen in Figs. 4.

\section{Now: current concepts in state-of-the-art quality control}

Diagnosis precedes the computer-assisted reconstruction of the orbit and is based on clinical findings and 3D imaging. ${ }^{24-28}$
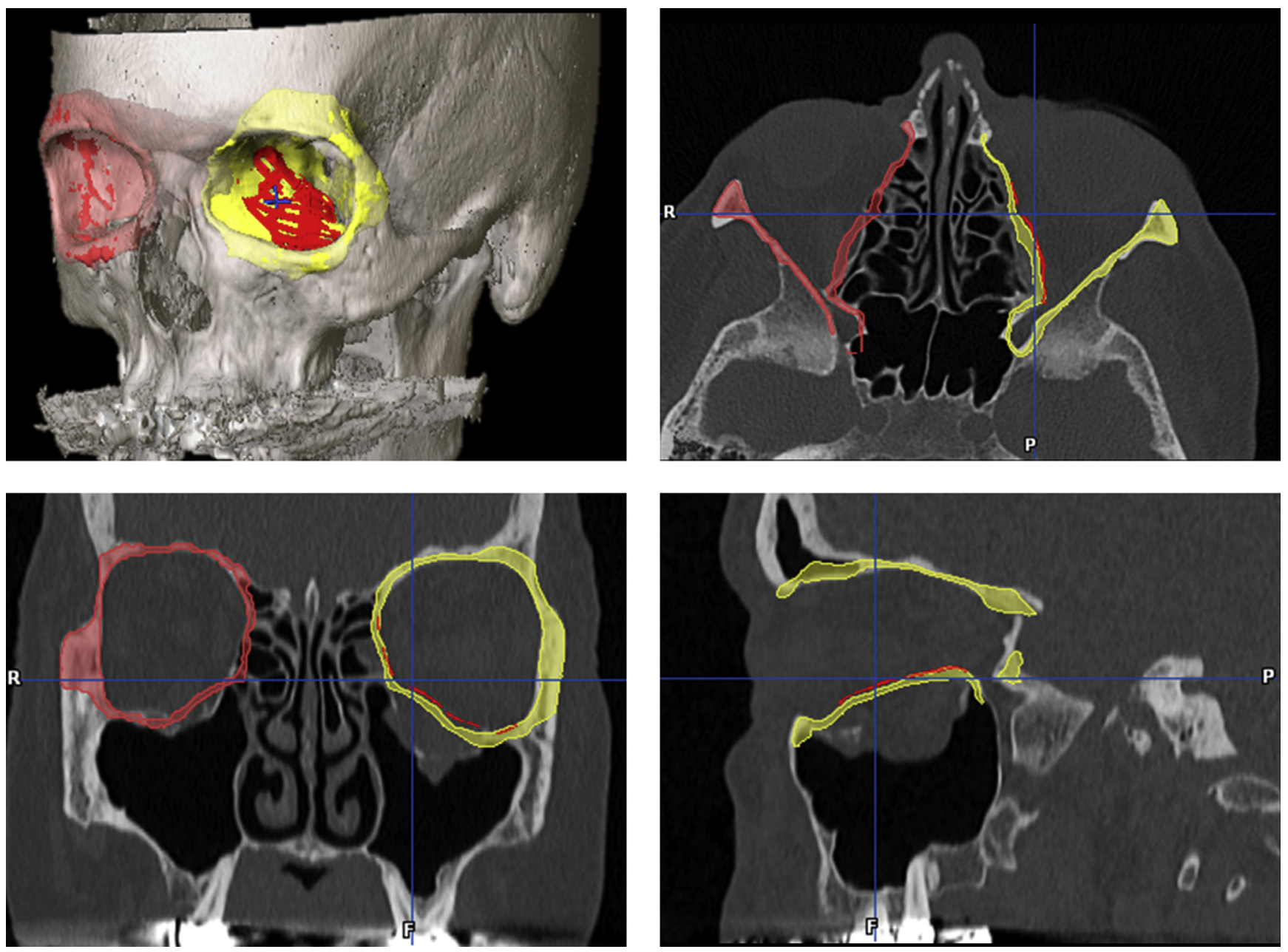

Fig. 5 Multiplanar view of the virtual reconstruction with a virtually placed patient-specific implant that was imported into the left orbit as an STL data set after industrial planning (red contour line). Virtual implant placement allows the accuracy of fit to be verified before the implant is manufactured. (Courtesy of Ruud Schreurs, MSc, Frank Wilde, MD, DDS, Alexander Schramm, MD, DDS, and Nils-Claudius Gellrich, MD, DDS.) 

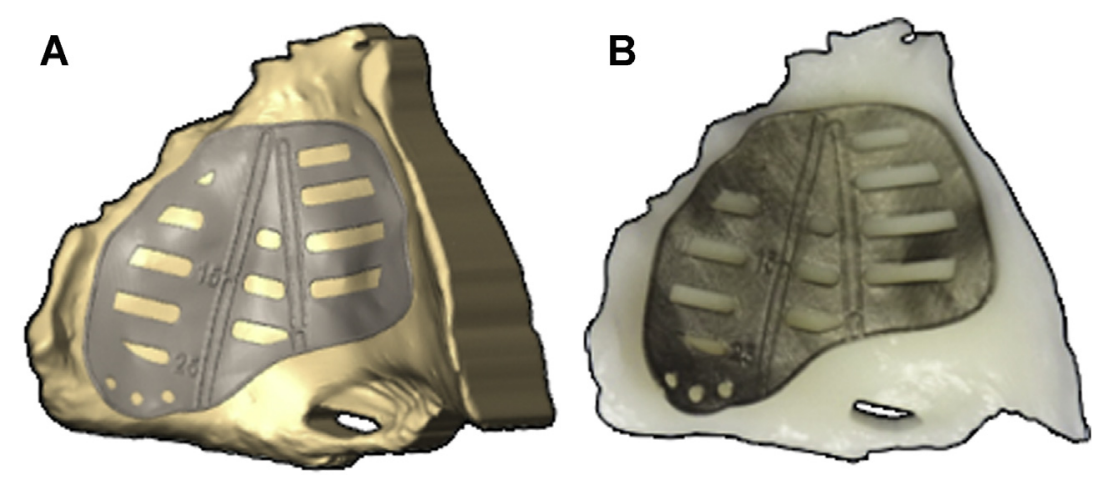

Fig. 6 Computer-aided design of the patient-specific implant $(A)$ and the selective laser-melted patient-specific implant corresponding to the design (B). (Courtesy of Ruud Schreurs, MSc, Frank Wilde, MD, DDS, Alexander Schramm, MD, DDS, and Nils-Claudius Gellrich, MD, DDS.)

As discussed in article,"Advanced Diagnostics and Three-dimensional Virtual Surgical Planning in Orbital Reconstruction", in this issue CT must be regarded as the gold standard for primary diagnosis preceding orbital reconstruction and should be preferred. It provides not only excellent resolution of bone structures but, unlike CBCT, also allows soft-tissue structures to be evaluated, especially with the use of contrast agent. ${ }^{28}$ In current concepts of intraoperative feedback workflows, virtual surgical planning and simulation of the reconstruction serves as the reference of the feedback obtained. The objective of surgical planning is to perform an ideal reconstruction based on a virtual model that matches the desired outcome of surgery. 1,7,29,30

Although discussed in greater detail in article,"Advanced Diagnostics and Three-dimensional Virtual Surgical Planning in Orbital Reconstruction", in this issue several important factors include that many different types of simulations may be performed without a loss of information, 2D and 3D images may be displayed in any plane before and during surgery, and virtual models (STL data) may be imported. ${ }^{1,31-33}$ These factors allow that standard implants, anatomically preformed implants, and patient-specific implants can be virtually positioned in the patient data set preoperatively and their shape, size, and fit can be assessed (Fig. 5). ${ }^{26,30,32}$ If a patient-specific implant is indicated for the orbital reconstruction, the next step on the ladder is to process virtual reconstruction STL data sets using computeraided design (CAD) software for planning patient-specific implants (Fig. 6A) and to directly produce these implants using computer-aided manufacturing (CAM) techniques such

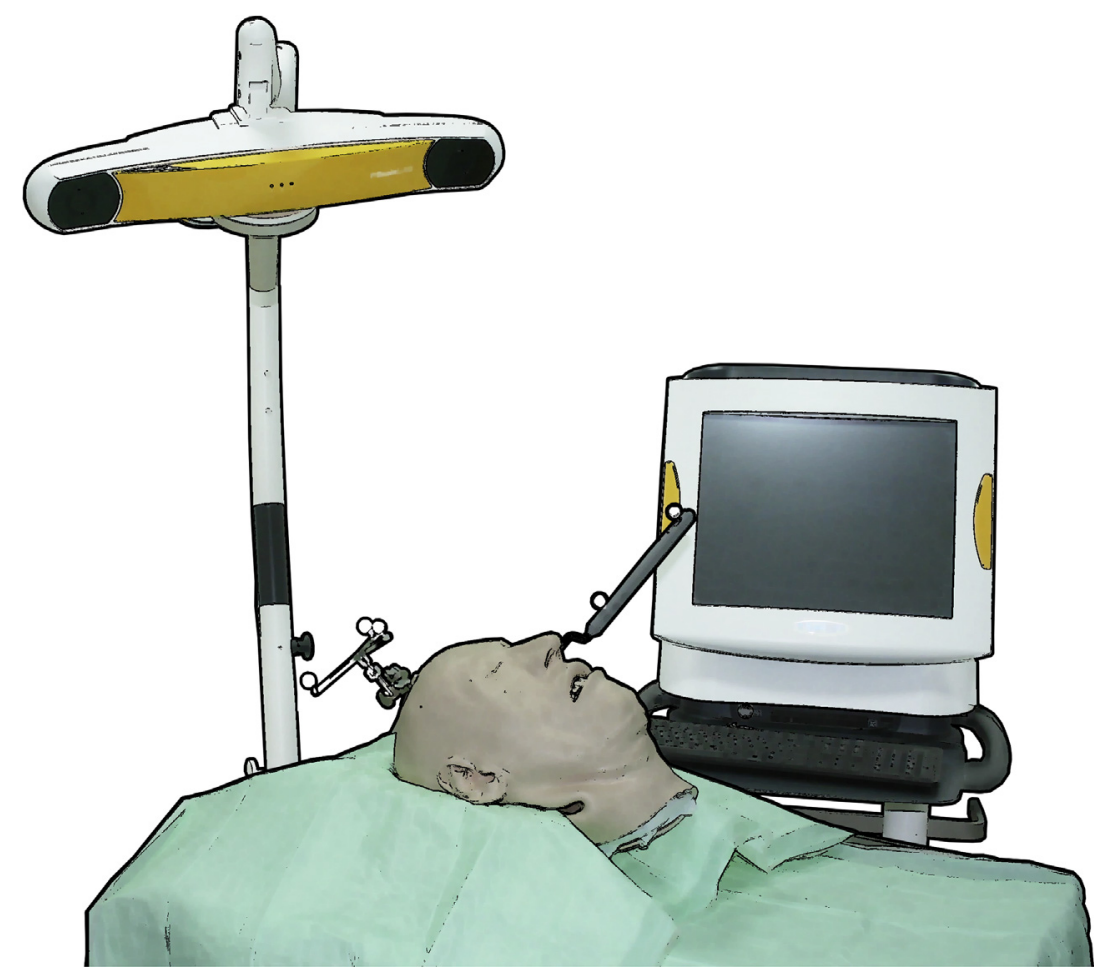

Fig. 7 Intraoperative setup of an indirect navigation system: camera with integrated LEDs, pointer with 2 reflective spheres, reference array with 3 reflective spheres attached to the calvaria, touch screen. (Courtesy of Ruud Schreurs, MSc, Frank Wilde, MD, DDS, Alexander Schramm, MD, DDS, and Nils-Claudius Gellrich, MD, DDS.) 

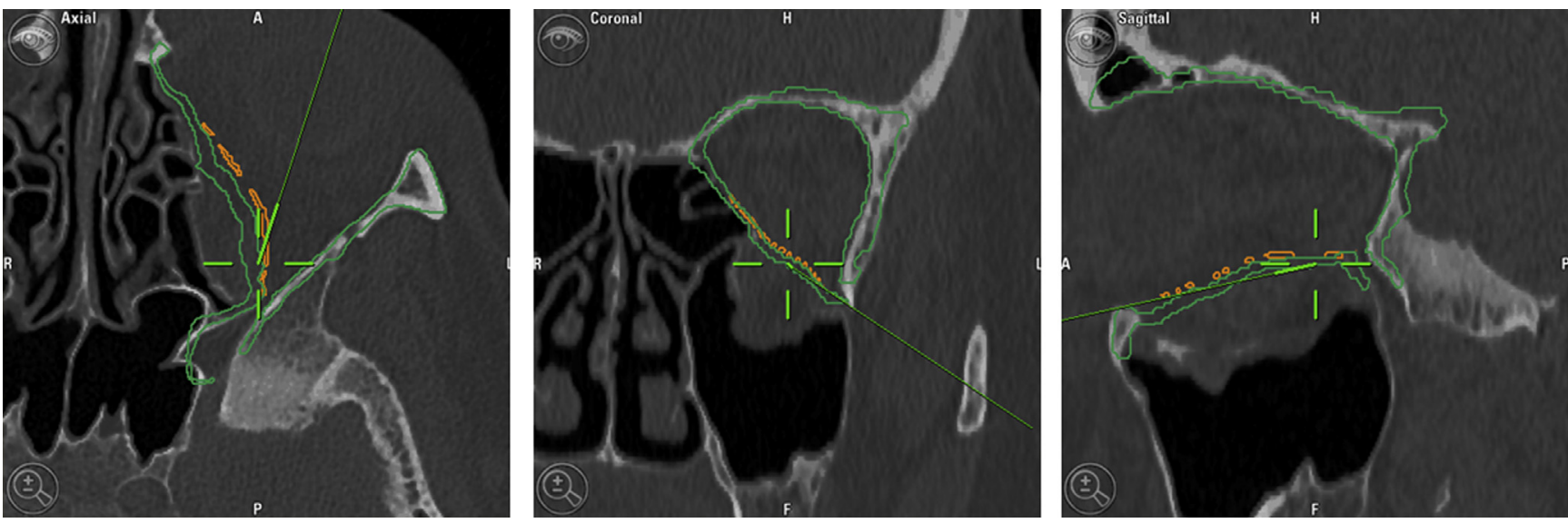

Fig. 8 Screenshots from multiplanar views on the navigation screen during surgery. The green line with the green cross represents the pointer in the orbit to control the position and fit of inserted implants during surgery. (Courtesy of Ruud Schreurs, MSC, Frank Wilde, MD, DDS, Alexander Schramm, MD, DDS, and Nils-Claudius Gellrich, MD, DDS.)

as selective laser melting (Fig. 6B). During the surgical procedure, surgeons have the difficult task of transferring the result of virtual computer-assisted planning to the surgical site as accurately as possible. For this purpose, they can use and combine a variety of different methods and devices, depending on the complexity of the task.

\section{Patient-specific implants}

Customized CAD-/CAM-fabricated implants are increasingly used for orbital reconstructions. Titanium mesh structures have proved their favorable material characteristics for orbital reconstructions. Selective laser melting of titanium powder allows high-precision titanium mesh structures of any complexity and geometry to be manufactured. Because it is now possible to obtain such patient-specific implants within 5 working days from planning to delivery, it has become possible to use such implants even for primary and not exclusively for secondary reconstructions. The aforementioned functionalization and preventive design parameters are incorporated as much as possible in the implant's design, so that not only "the perfect lid could be manufactured for an individual pot" but also "the perfect lid could sit in the perfect position." The exact fit of the implant, the use of surgical guides, the incorporation of "old" screw hole positions from the primary reconstruction (in secondary cases), and the use of navigation markers or rulers all aid in achieving this goal. ${ }^{23,34}$ The challenge to obtain a correct postoperative result without the use of these implants is definitely higher. Apart from the orbitwhich pioneered these implant developments with much higher demands and clinical relevance-any other implant used in craniomaxillofacial surgery or even the human body profits from these advances in orbital surgery.

\section{Intraoperative navigation}

Nowadays, intraoperative navigation with modern infraredbased navigation systems has become an integral part of computer-assisted maxillofacial reconstruction. ${ }^{1,7}$ Before the start of the surgical procedure, the patient in the operation room must be "fused" with the image data set that is displayed on the screen. This process is known as registration and is based on reference points that must be clearly identifiable and reproducible both on the patient and in the image data set. Because the patient's position must be permanently tracked because patient movements during surgery cannot be prevented or may even be necessary during the procedure, a reference array is attached to the patient's head, which allows the navigation system to register all patient movements without limiting head mobility (see Fig. 7). 1,7,32

Once the patient is registered with the DICOM set, the tip of the pointer can be visualized in the image volume on the screen of the navigation system, which enables surgeons to verify the realignment of bone fragments and the position and fit of inserted implants during surgery and to make any necessary changes without patient exposure to ionizing radiation. In this process, the virtual reconstruction that has been made during the planning phase serves as an intraoperative template that helps surgeons align bone fragments and/or place implants (Fig. 8). 1,7,29,30 The direction of the navigation rulers, or the position of navigation markers, allows easier interpretation of the acquired implant position by the surgeon. ${ }^{23,35}$

\section{Intraoperative imaging}

Although functionalized implants and intraoperative navigation can provide usable information during reconstruction, the surgical outcome in orbital surgery should always be validated and documented in 3D by the use of CT or CBCT. Although intraoperative navigation can already provide relatively precise information about the position of osteosynthesis material and the repositioning of bone fragments, it cannot completely replace a radiological examination, because navigation can never visualize the entire surgical site. It is recommendable that such a radiological validation should be performed during the surgical procedure immediately after reconstruction, which allows necessary modifications such as incorrect positions of bone fragments and implants to be made during the surgical procedure. ${ }^{27,30,32}$ CBCT technology offers several advantages that make this modality superior. CBCT systems are available as mobile, ceiling-suspended, or floor-mounted 3D Carm systems for use in the operating room or as small compact CBCT systems for intraoperative use (Fig. 9). ${ }^{32}$ The systems produce image series for multiplanar visualization in the axial, 


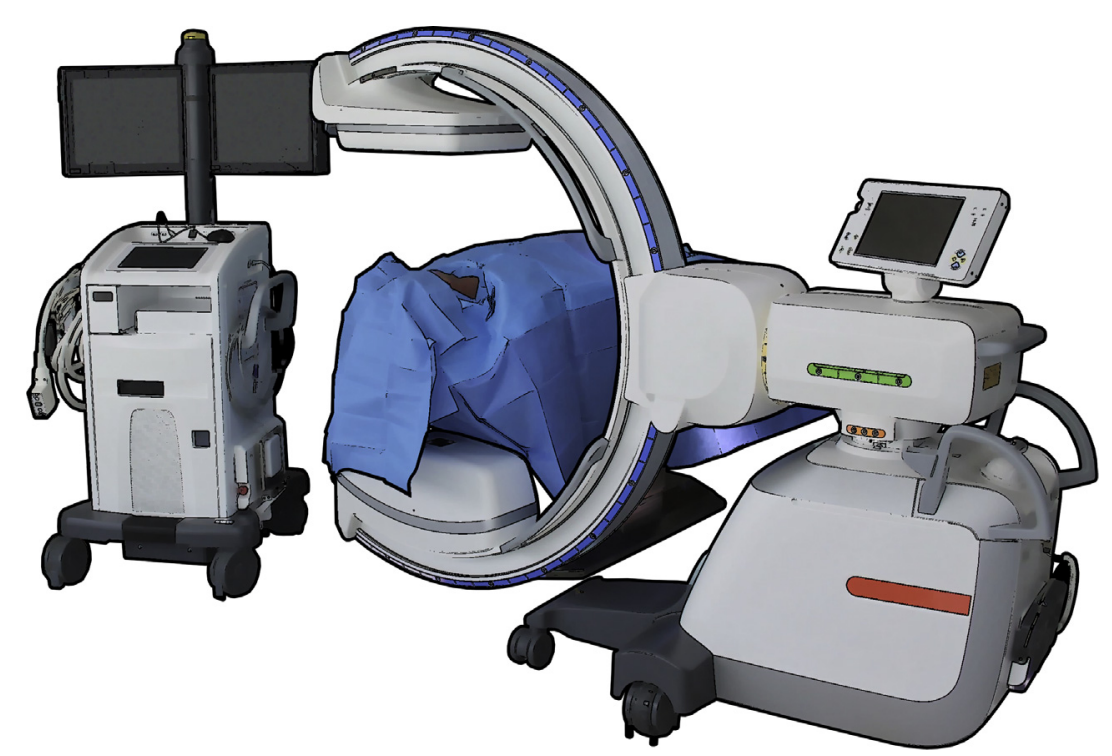

Fig. 9 Mobile 3D C-arm device for intraoperative imaging in action. (Courtesy of Ruud Schreurs, MSc, Frank Wilde, MD, DDS, Alexander Schramm, MD, DDS, and Nils-Claudius Gellrich, MD, DDS.)

coronal, and sagittal planes and thus allow hard tissues to be imaged during surgery. Images that are produced by these systems are nowadays of such good quality that additional postoperative imaging is usually not required anymore. ${ }^{27}$

The purpose of intraoperative and postoperative 3D imaging is not only to perform a surgical procedure under radiological control but also to provide data sets for computer-assisted fusion with preoperative image data sets and especially with preoperative computer planning. Image fusion allows differences between preoperative planning and surgical outcome to be visualized and, if required, to be quantitatively evaluated within the scope of quality assurance (Fig. 10).

\section{Wow: the future of orbital reconstruction}

Technological innovation will affect the utilization of all of the abovementioned technologies for intraoperative feedback and even add novel methods of obtaining feedback during surgery. Separate feedback modalities are already combined into a symbiotic feedback system: the aforementioned functionalization of a patient-specific implant design allows it to be equipped with navigation rulers and markers to optimize navigation feedback (see Fig. 4). ${ }^{23,36}$ The unique fit and addition of rulers and markers in the design is an example of 2 combined feedback mechanisms that allow improved feedback
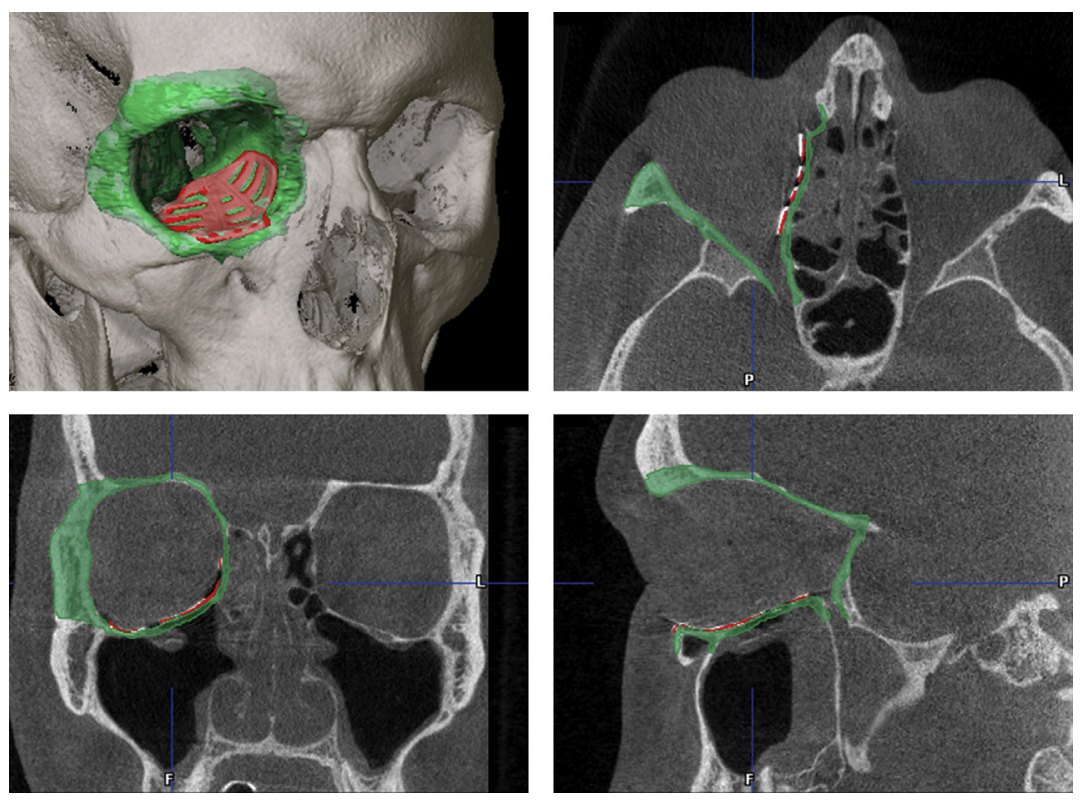

Fig. 10 Multiplanar view of intraoperative 3D imaging with superimposition of virtual planning. The green contour line shows the virtual reconstruction of the orbit and the red contour the planned implant. The image shows good fit of the radio-opaque implant in accordance with virtual planning. (Courtesy of Ruud Schreurs, MSc, Frank Wilde, MD, DDS, Alexander Schramm, MD, DDS, and Nils-Claudius Gellrich, MD, DDS.) 


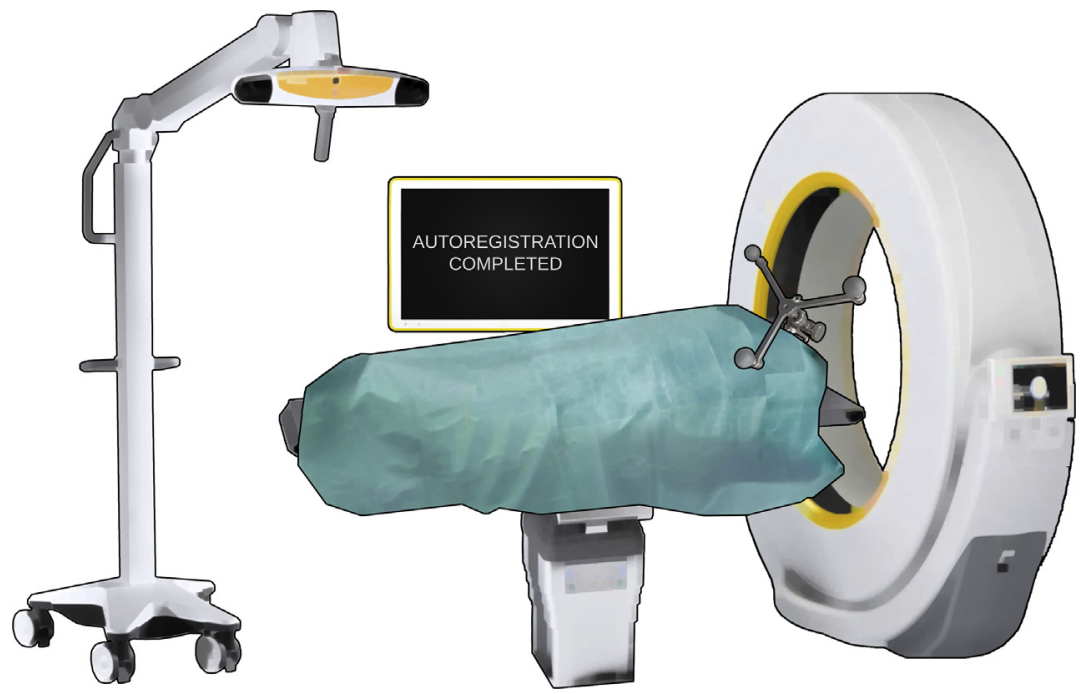

Fig. 11 Autoregistration setup. Intraoperative imaging is combined with the registration procedure for navigation, allowing (re-) registration in the operation room without the need for user intervention. (Courtesy of Ruud Schreurs, MSc, Frank Wilde, MD, DDS, Alexander Schramm, MD, DDS, and Nils-Claudius Gellrich, MD, DDS.)

intraoperatively. Another example is the fact that intraoperative imaging can be transferred to the preoperative planning modality automatically to generate instant feedback on the reconstruction result ${ }^{37-39}$ and may even provide (re-) registration if used in combination with intraoperative navigation (Fig. 11). Registration of the patient to the virtual surgical plan can now be performed without the need for user intervention. Still, improvements to the already comprehensive feedback are possible in several areas, such as preoperative design, preoperative simulation, intraoperative visualization, and intraoperative control.

The design of a patient-specific implant is nowadays performed largely manually: a designer from a third-party company designs the implant in consultation with the surgeon, or in specific cases the design is performed in-house by a clinical technician or the surgeon. In every scenario, the design is based on the individual patient's characteristics but influenced by experience and preference of designer and surgeon. Design considerations such as implant's size, compelling fit, and feedback mechanisms incorporated are operator dependent, and the quality of intraoperative feedback obtained from the shape and fit of the patient-specific implant is affected by previous experience in patient-specific implant design. An overview of the design workflow is provided in Fig. 12A. The use of artificial intelligence in the preoperative planning process could induce automation in the patient-specific implant design process, while bearing optimal intra-operative feedback in mind (Fig. 12B), and this could save time in the planning process, perfect the compelling fit, and make individualized treatment more accessible to surgeons without previous experience in patient-specific implant design.

Obtaining experience in orbital reconstruction is difficult in general, because of the keyhole access and limited exposure of the surgical field. The intraoperative feedback modalities may aid in the understanding of the procedure, but they are no substitute for hands-on learning experience. Preoperative surgical simulation could provide a possible solution. ${ }^{40}$ In current state-of-the-art simulation, a walk-through of the complete surgery is performed on a 3D-printed model consisting of several components, which simulate the tactile feedback of anatomic structures encountered. ${ }^{41}$ The virtual model is seen in Fig. 13A, and the physical components are seen in Fig. 13B. Intraoperative navigation for quality control in the simulation setting makes the training surgeon familiar with its use and interpretation (Fig. 13C). Simulation could also benefit experienced surgeons, for complex conditions or procedures. Currently, physical models are used, because virtual simulation is associated with high cost and difficulty to simulate invasive procedures. If these drawbacks are overcome, virtual simulation obviates production time and cost and has the potential to simulate patient-specific surgeries (repetitively) and be incorporated in the computer-assisted surgery workflow.

Visualization during surgery could also benefit from further developments. Although frequently compared with car navigation, intraoperative navigation is not nearly as intuitive. Feedback is provided to review the acquired position, rather than real-time during positioning, and interpretation of implant position from the navigation pointer's position is left to the surgeon. Real-time, intuitive feedback during positioning (similar to car navigation) requires the implant to be tracked at all times and its position expressed relative to the target position. A navigation-compatible positioning instrument for orbital reconstruction has been proposed that meets these requirements (Fig. 14), and software has been developed to provide real-time instructions on positioning, both visually and quantitatively. ${ }^{42}$ Positioning with the instrument and the realtime navigation feedback obtained are visualized in Fig. 15. The effect on implant position has been established in preclinical testing (see article, "Advanced Concepts of Orbital Reconstruction: A Unique Attempt to Scientifically Evaluate Individual Techniques in Reconstruction of Large Orbital Defects," in this issue. ..$^{43}$

Current methods using visual feedback require the surgeon to evaluate 3D information on a 2D screen and switch focus between surgical site and monitor. Augmented reality possesses the possibility to tackle both problems by enhancing the surgeon's view of the surgical site with a virtual overlay. ${ }^{44-47}$ The information presented in this overlay could be the virtual planning: in Fig. 16A, the surgeon's view of the real world is seen; the virtual layer in Fig. 16B contains objects from the 


\section{A}

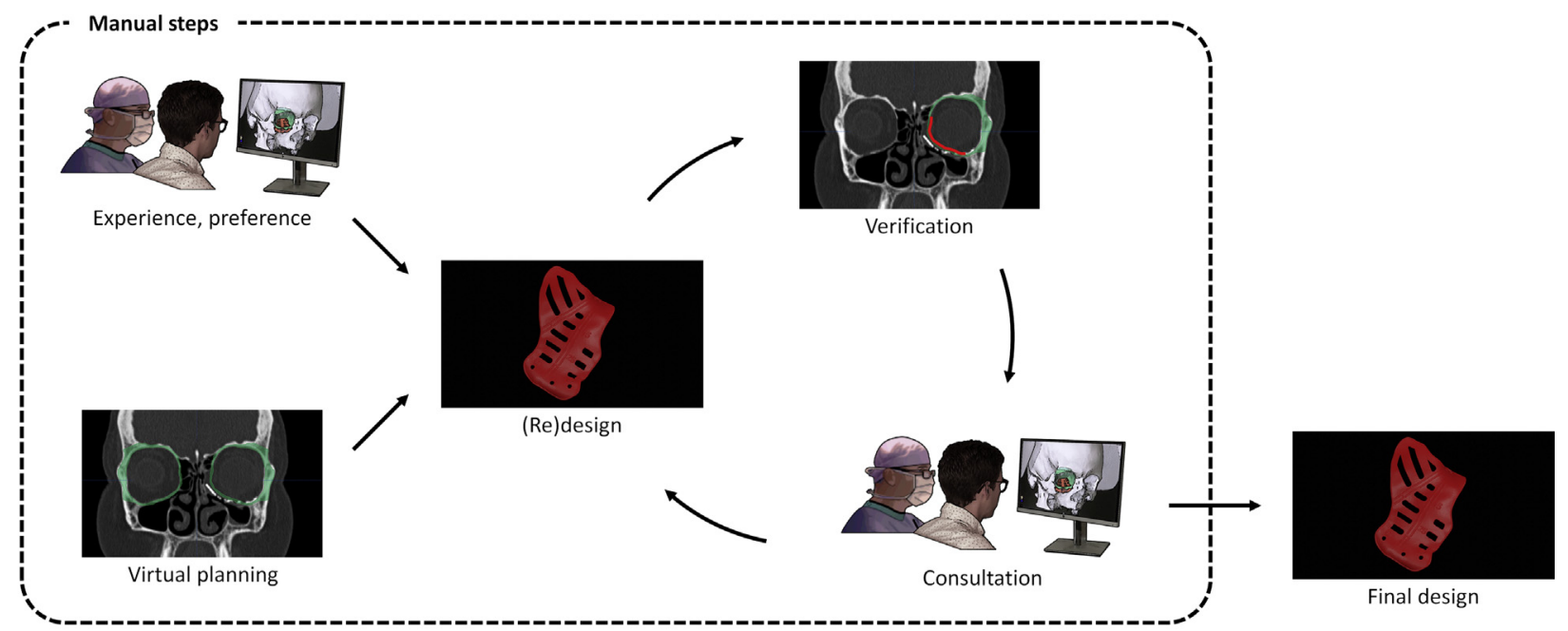

B

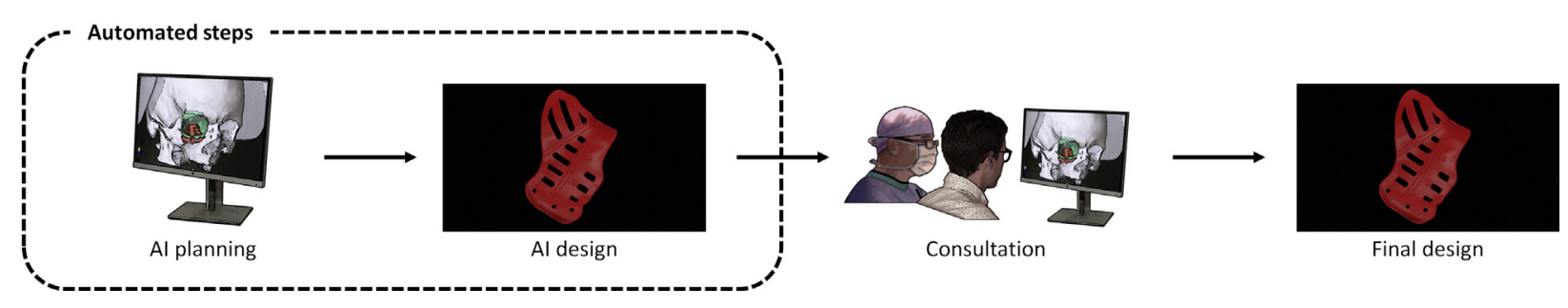

Fig. 12 Visualization of current PSI design workflow $(A)$, with much of the design choices based on experience and preference of the surgeon and designer. Many manual steps need to be completed in order to obtain the final PSI design. In (B), a proposed Al-supported workflow is visualized. The planning and preliminary design is performed largely automatically, saving time and making PSI design more accessible to surgeons without previous experience in PSI treatment. Al, artificial intelligence; PSI, patient-specific implant. (Courtesy of Ruud Schreurs, MSc, Frank Wilde, MD, DDS, Alexander Schramm, MD, DDS, and Nils-Claudius Gellrich, MD, DDS.)
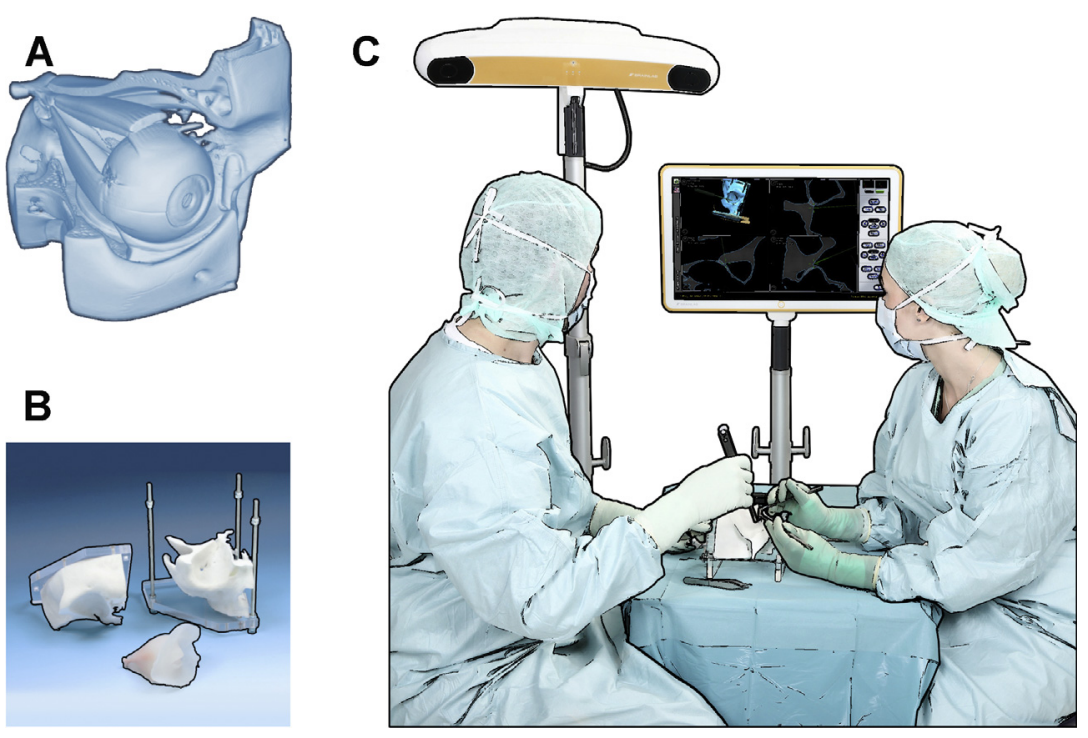

Fig. 13 Surgical simulation of orbital reconstruction. The virtual model of the orbit and orbital contents is visualized in $(A)$. Physical models based on the virtual model are generated through 3D printing; the individual components consist of different materials to simulate tactile feedback during surgery $(B)$. The simulation is compatible with intraoperative navigation, which allows the surgeon to get familiar with the interpretation of the feedback the system provides. The complete setup is seen in (C). (Courtesy of Ruud Schreurs, MSc, Frank Wilde, MD, DDS, Alexander Schramm, MD, DDS, and Nils-Claudius Gellrich, MD, DDS.) 


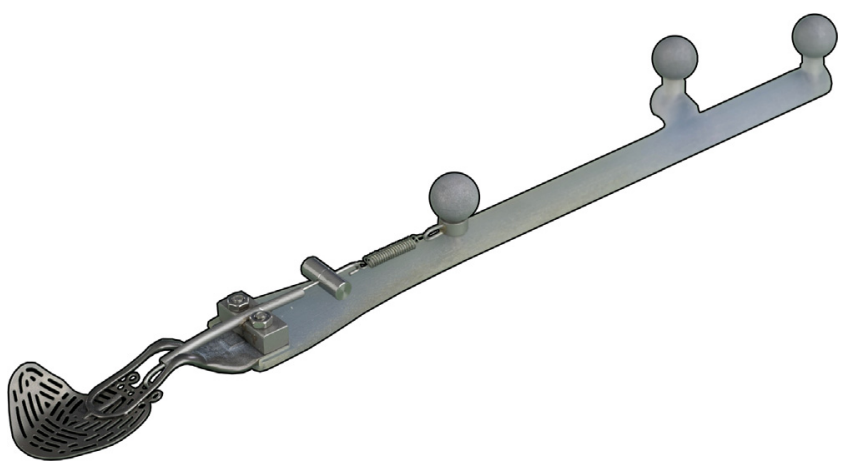

Fig. 14 Navigation-compatible positioning instrument. The orbital implant can be attached to the instrument in a unique way (and detached after fixation); the position of the orbital implant can be calculated from the position of the navigation feedback the instrument generates. (Courtesy of Ruud Schreurs, MSc, Frank Wilde, MD, DDS, Alexander Schramm, MD, DDS, and NilsClaudius Gellrich, MD, DDS.)

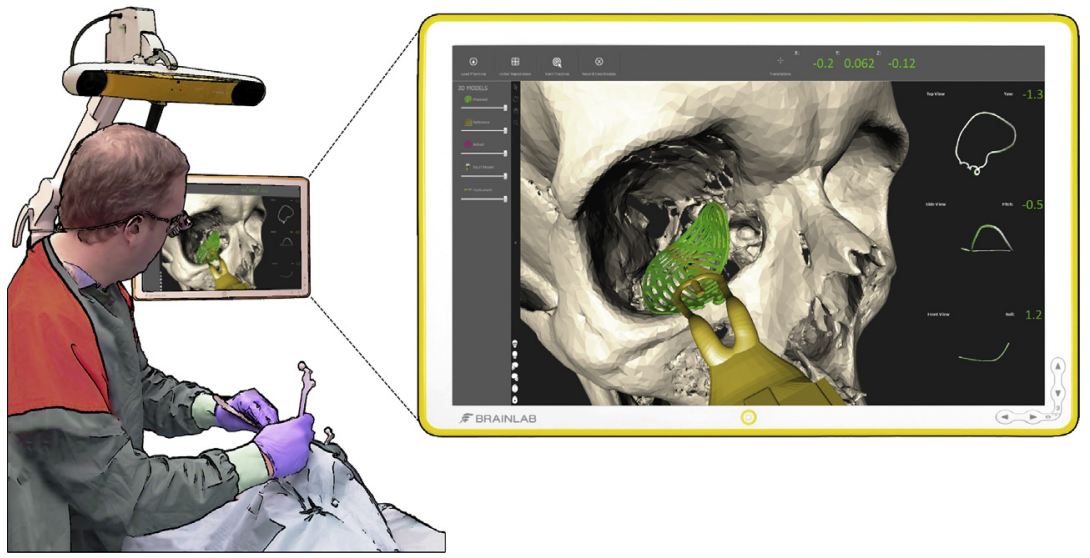

Fig. 15 Real-time navigation setup and feedback. The orbital implant is tracked through the instrument, which the surgeon uses to position the orbital implant. Real-time feedback consists of a 3D view of the planned and current position of the implant; the color changes according to the current deviation. Separate views representing a top view, side view, and frontal view of the implant outline are added for detailed positioning information. Quantitative feedback on rotation and translation is provided next to the separate views and top, respectively. (Courtesy of Ruud Schreurs, MSc, Frank Wilde, MD, DDS, Alexander Schramm, MD, DDS, and Nils-Claudius Gellrich, MD, DDS.)

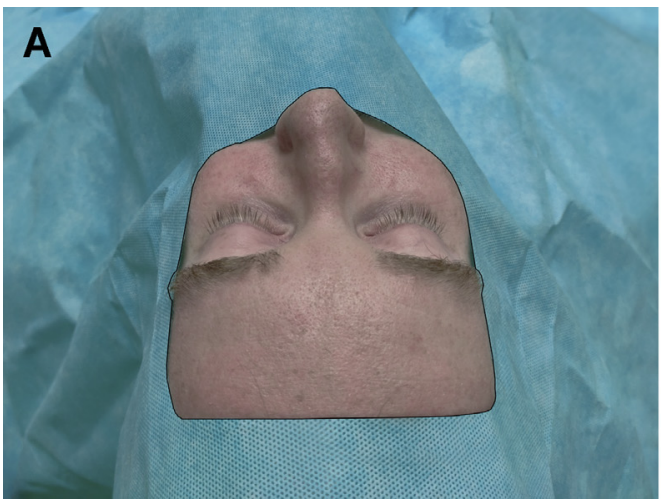

B
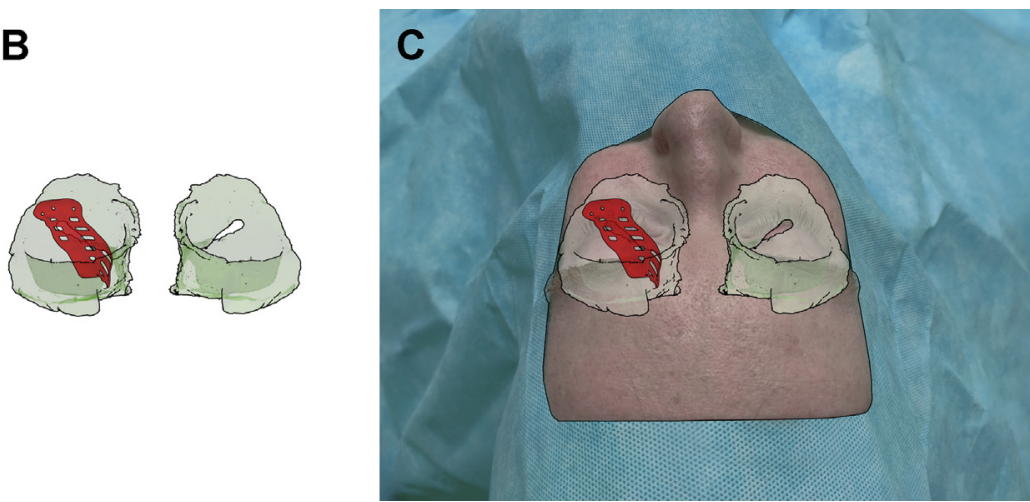

Fig. 16 Principle of augmented reality. The real world, as seen by the surgeon, is seen in $(A)$. In $(B)$, the virtual layer to be augmented on the real-world view is shown, which corresponds to the planned objects in the virtual surgical planning. In $(C)$, the augmented reality view is shown: the virtual layer is added to the real world for a combined view. (Courtesy of Ruud Schreurs, MSc, Frank Wilde, MD, DDS, Alexander Schramm, MD, DDS, and Nils-Claudius Gellrich, MD, DDS.) 
planning. In Fig. 16C, the augmented view of the surgeon is seen. Even more valuable feedback may become available through combination with real-time navigation: a see-through view of the orbit with the planned and current implant position could be added to the operator's view.

In a more distant future, robotic-assisted orbital reconstruction may become available. Several approaches could be imagined, ranging from simple tasks such as aiding in fixation by stabilizing the acquired implant position to more complex methods, such as movement constraints for the surgeon to keep clear of vital anatomic structures and minimize the amount of iatrogenic damage. ${ }^{48,49}$ Fully autonomous implant insertion may even be feasible from a technical point of view, but this may be constrained by medicolegal issues. The key to successful implementation of any of these futuristic technologies in orbital reconstruction may be the same as the strategy seen in current intraoperative feedback methods: combining techniques to use their respective strengths and cover the individual frailties.

\section{CLINICS CARE POINTS}

- Patient-specific implants hold the highest potential for optimal reconstruction of an orbital defect. A design tailored to the defect and surrounding anatomy, and functionalization of the implant for combination with other feedback techniques, ensure an optimal implant that leads to an optimal reconstruction.

- Intraoperative navigation has become an integral part of computer-assisted maxillofacial reconstruction. It is able to provide feedback to improve predictability during orbital reconstruction.

- The surgical outcome in orbital reconstruction should always be validated and documented. If imaging is performed intraoperatively, necessary modifications may be made during the surgical procedure and revision surgeries may be prevented.

\section{Disclosure}

The authors have nothing to disclose.

\section{References}

1. Schramm A, Gellrich NC. Intraoperative navigation und computerassistierte Chirurgie. Schwenzer N Und Ehrenfeld M ZahnMund-Kieferheilkunde. Mund Kiefer Gesichtschir 2011;4:479-99.

2. Gellrich N-C, Schramm A, Hammer B, et al. Computer-assisted secondary reconstruction of unilateral posttraumatic orbital deformity. Plast Reconstr Surg 2002;110(6):1417-29.

3. Metzger MC, Bittermann G, Dannenberg L, et al. Design and development of a virtual anatomic atlas of the human skull for automatic segmentation in computer-assisted surgery, preoperative planning, and navigation. Int J Comput Assist Radiol Surg 2013; 8(5):691-702.

4. Horsley V, Clarke RH. The structure and functions of the cerebellum examined by a new method. Brain 1908;31(1):45-124.

5. Schurr PH, Merrington WR. The Horsley-Clarke stereotaxic apparatus. Br J Surg 1978;65(1):33-6.

6. Jensen RL, Stone JL, Hayne RA. Introduction of the human HorsleyClarke stereotactic frame. Neurosurgery 1996;38(3):563-7.

7. Schramm A, Gellrich N-C, Schmelzeisen R. Navigational surgery of the facial skeleton. Springer Science \& Business Media; 2007.
8. Spiegel EA, Wycis HT, Marks M, et al. Stereotaxic apparatus for operations on the human brain. Science 1947;106(2754): 349-50.

9. Watanabe E, Watanabe T, Manaka S, et al. Three-dimensional digitizer (neuronavigator): new equipment for computed tomography-guided stereotaxic surgery. Surg Neurol 1987;27(6):543-7.

10. Shalit MN, Israeli Y, Matz S, et al. Intra-operative computerized axial tomography. Surg Neurol 1979;11(5):382-4.

11. Lunsford LD, Parrish R, Albright L. Intraoperative imaging with a therapeutic computed tomographic scanner. Neurosurgery 1984; 15(4):559-61.

12. Schichor C, Terpolilli N, Thorsteinsdottir J, et al. Intraoperative computed tomography in cranial neurosurgery. Neurosurg Clin $\mathrm{N}$ Am 2017;28(4):595-602.

13. Nimsky C, Carl B. Historical, current, and future intraoperative imaging modalities. Neurosurg Clin N Am 2017;28(4):453-64.

14. Stanley RB. Use of intraoperative computed tomography during repair of orbitozygomatic fractures. Arch Facial Plast Surg 1999; 1(1):19-24.

15. Doerfler H-M, Huempfner-Hierl H, Kruber D, et al. Template-based orbital wall fracture treatment using statistical shape analysis. J Oral Maxillofac Surg 2017;75(7):1475-e1.

16. Kim YC, Jeong WS, Park T, et al. The accuracy of patient specific implant prebented with 3D-printed rapid prototype model for orbital wall reconstruction. J Craniomaxillofac Surg 2017;45(6):928-36.

17. Metzger MC, Schön R, Tetzlaf R, et al. Topographical CT-data analysis of the human orbital floor. Int J Oral Maxillofac Surg 2007; 36(1):45-53.

18. Bell RB. Computer planning and intraoperative navigation in cranio-maxillofacial surgery. Oral Maxillofac Surg Clin North Am 2010; 22(1):135-56.

19. Metzger MC, Schmelzeisen R. Craniomaxillofacial Implants Based on 3D Modeling. Digital Technologies in Craniomaxillofacial Surgery. Springer; 2018. p. 167-73.

20. Strong EB, Fuller SC, Wiley DF, et al. Preformed vs intraoperative bending of titanium mesh for orbital reconstruction. Otolaryngol Head Neck Surg 2013;149(1):60-6.

21. Bittermann G, Metzger MC, Schlager S, et al. Orbital reconstruction: prefabricated implants, data transfer, and revision surgery. Facial Plast Surg 2014;30(05):554-60.

22. Hierl T, Kruber D, Doerfler H-M, et al. Computer-aided versus conventional planning in orbital traumatology using preformed meshes: development of a new workflow. J Oral Maxillofac Surg 2019;77(8):1663-72.

23. Rana M, Chui CHK, Wagner M, et al. Increasing the accuracy of orbital reconstruction with selective laser-melted patient-specific implants combined with intraoperative navigation. J Oral Maxillofac Surg 2015;73(6):1113-8.

24. Manson PN, Markowitz B, Mirvis S, et al. Toward CT-based facial fracture treatment. Plast Reconstr Surg 1990;85(2):202-12.

25. Tanrikulu R, Erol B. Comparison of computed tomography with conventional radiography for midfacial fractures. Dentomaxillofac Radiol 2001;30(3):141-6.

26. Wilde F, Hilbert J, Kamer L, et al. The combination of automatic segmentation, preformed implants, intraoperative navigation and intraoperative imaging in primary orbital wall reconstruction: description of a new method. Int J Comput Assist Radiol Surg 2009;4:134.

27. Wilde F, Lorenz K, Ebner A-K, et al. Intraoperative imaging with a 3D C-arm system after zygomatico-orbital complex fracture reduction. J Oral Maxillofac Surg 2013;71(5):894-910.

28. Wikner J, Riecke B, Gröbe A, et al. Imaging of the midfacial and orbital trauma. Facial Plast Surg 2014;30(05):528-36.

29. Hohlweg-Majert B, Schön R, Schmelzeisen R, et al. Navigational maxillofacial surgery using virtual models. World J Surg 2005; 29(12):1530-8.

30. Schramm A, Wilde F. Die computergestützte Gesichtsschädelrekonstruktion. HNO 2011;59(8):800.

31. Markiewicz MR, Bell RB. Modern concepts in computer-assisted craniomaxillofacial reconstruction. Curr Opin Otolaryngol Head Neck Surg 2011;19(4):295-301. 
32. Wilde F, Schramm A. Intraoperative imaging in orbital and midface reconstruction. Facial Plast Surg 2014;30(05):545-53.

33. Parthasarathy J. 3D modeling, custom implants and its future perspectives in craniofacial surgery. Ann Maxillofac Surg 2014; 4(1):9.

34. Schreurs R, Dubois L, Becking AG, et al. The orbit first! A novel surgical treatment protocol for secondary orbitozygomatic reconstruction. J Craniomaxillofac Surg 2017;45(7). https: //doi.org/10.1016/j.jcms.2017.03.026.

35. Dubois L, Essig $\mathrm{H}$, Schreurs R, et al. Predictability in orbital reconstruction. A human cadaver study, part III: implant-oriented navigation for optimized reconstruction. J Craniomaxillofac Surg 2015;43(10):2050-6.

36. Gander T, Essig H, Metzler P, et al. Patient specific implants (PSI) in reconstruction of orbital floor and wall fractures. J Craniomaxillofac Surg 2015;43(1):126-30.

37. Grauvogel TD, Becker C, Hassepass F, et al. Comparison of 3D Carm-based registration to conventional pair-point registration regarding navigation accuracy in ENT surgery. Otolaryngol Head Neck Surg 2015;152(2):266-71.

38. Carl B, Bopp M, Saß B, et al. Intraoperative computed tomography as reliable navigation registration device in 200 cranial procedures. Acta Neurochir (Wien) 2018;160(9):1681-9.

39. Carl B, Bopp M, Saß B, et al. Reliable navigation registration in cranial and spine surgery based on intraoperative computed tomography. Neurosurg Focus 2019;47(6):E11.
40. Selzer DJ. Overview of Simulation in Surgery. Comprehensive Healthcare Simulation: Surgery and Surgical Subspecialties. Springer; 2019. p. 13-23.

41. Lichtenstein JT, Zeller AN, Lemound J, et al. 3D-printed simulation device for orbital surgery. J Surg Educ 2017;74(1):2-8.

42. Schreurs R, Dubois L, Becking AG, et al. Implant-oriented navigation in orbital reconstruction. Part 1: technique and accuracy study. Int J Oral Maxillofac Surg 2017;47(3):395-402.

43. Schreurs R, Dubois L, Ho J, et al. Implant-oriented navigation in orbital reconstruction part II: preclinical cadaver study. Int J Oral Maxillofac Surg 2019;49(5):678-85.

44. Vigh B, Müller S, Ristow 0 , et al. The use of a head-mounted display in oral implantology: a feasibility study. Int J Comput Assist Radiol Surg 2014;9(1):71-8.

45. Wang J, Suenaga $\mathrm{H}$, Yang $\mathrm{L}$, et al. Video see-through augmented reality for oral and maxillofacial surgery. Int J Med Robot 2017; 13(2):e1754.

46. Meulstee JW, Nijsink J, Schreurs R, et al. Toward holographicguided surgery. Surg Innov 2018;26(1). 1553350618799552.

47. Wang J, Shen Y, Yang S. A practical marker-less image registration method for augmented reality oral and maxillofacial surgery. Int $\mathrm{J}$ Comput Assist Radiol Surg 2019;14(5):763-73.

48. Davies B. A review of robotics in surgery. Proc Inst Mech Eng $\mathrm{H}$. 2000;214(1):129-40.

49. Camarillo DB, Krummel TM, Salisbury JK Jr. Robotic technology in surgery: past, present, and future. Am J Surg 2004;188(4):2-15. 